\title{
SPATIAL DISCRETIZATION OF THE DUSTY GAS EQUATIONS
}

\author{
BY \\ DAVID GOTTLIEB (Brown University, Division of Applied Mathematics, 182 George Street, Box \\ F, Providence, RI 02912) \\ AND
}

ROGER TEMAM (The Institute for Scientific Computing and Applied Mathematics, Indiana University, Bloomington, IN 47405 and Laboratoire d'Analyse Numérique, Université Paris-Sud, Bâtiment 425, 91405 Orsay, France)

\begin{abstract}
In this article we consider a semi-discretization of the dusty gas equations corresponding to a spatial discretization by finite differences or pseudo-spectral methods and discuss the stability and well-posedness of the corresponding system of ordinary equations.

We will present elsewhere the study of the fully discretized equations (discretization in space and time) as well as some elements of the mathematical theory of the equations (existence and uniqueness of solutions).
\end{abstract}

1. Introduction. The dusty gas equations are used to describe infiltration processes needed for the manufacturing of a variety of materials. In those techniques a fluid phase (i.e., a gas or a liquid) is transported into a porous structure, where it then reacts to form a solid product. These methods are particularly important for producing composite materials, where the initial porous form is composed of the reinforcement phase (i.e., fibers, whiskers, or particles) and infiltration produces the matrix (see, e.g., [2] and the references therein).

For the mathematical modelling we consider the domain $\Omega$ filled by the mixtures, and denote by $C_{1}, \ldots, C_{s}$ the concentrations of these mixtures that are functions of space and time, $C_{i}=C_{i}(x, t)$. These concentrations are governed by the equations (2.2)(2.5). Instead of introducing the thermohydraulics equations, we assume for the sake of simplicity that the pressure $p$ and the temperature $T$ are known (see (2.1)). The equations for the $C_{i}$ are supplemented by boundary conditions, and, for more generality, we consider here three different boundary conditions on $\partial \Omega$, namely the Dirichlet, Neumann, and space-periodicity boundary conditions.

Equations (2.2)-(2.5) are classical equations of chemical dynamics and they appear in other areas of sciences, e.g., in combustion [9]. These equations are highly nonlinear and,

Received September, 2002.

2000 Mathematics Subject Classification. Primary 65N12, 76T15, 35Q35. 
to the best of our knowledge, much remains to be done for their mathematical theory, on questions of existence and uniqueness of solutions. ${ }^{1}$ Because of the practical importance of these equations, we nevertheless tackle some issues concerning their discretization and numerical analysis here. Namely, we consider the space discretizations of these equations by either finite differences or spectral methods (Fourier or Legendre, depending on the type of boundary conditions used). We describe very precisely the corresponding discretizations of the equations leading to systems of ordinary differential equations. We then establish estimates on the solutions of these equations which, on one hand, prove existence for all time and, on the other hand, prove the stability of the discretization procedures (stability in the sense of numerical analysis). To the best of our knowledge, this is the first time that such a study is conducted for such highly nonlinear equations.

In a following subsequent article [3], we will study the one-dimensional case in more detail. We also hope to address subsequently the question of space and time discretizations which should hinge on further new tools.

2. Presentation of the problem. We consider $S$ different chemical species contained in a region $\Omega \subset \mathbb{R}^{d}, d=2$ or 3 . We denote by $M_{i}$ the molecular mass of species $i$ and by $C_{i}$ its molecular concentration. The total number of molecules of species $i$ per unit spatial volume at $(x, t)$ is denoted by $\nu_{i}=\nu_{i}(x, t)$ and $C_{i}=\nu_{i} \mathcal{A}$, where $\mathcal{A}$ is the Avogadro number; the density $\rho_{i}$ of species $i$ (mass per unit volume) is $\rho_{i}=M_{i} \nu_{i}=M_{i} C_{i} \mathcal{A}$ and $\rho=\sum_{i=1}^{S} \rho_{i}$ is the density of the mixture.

We denote by $p=p(x, t)$ and $T=T(x, t)$ the pressure and temperature of the mixture which are assumed to be given and satisfy

$$
\begin{aligned}
& 0 \leq \underline{p} \leq p(x, t) \leq \bar{p}, \\
& 0 \leq \underline{T} \leq T(x, t) \leq \bar{T}, \quad \forall x \in \Omega, \quad \forall t>0 .
\end{aligned}
$$

Let $N_{i}$ denote the flux vector of species $i$, so that $N_{i} / C_{i}$ is its relative velocity (velocity relative to the mixture). We then obtain the equation governing the motion of the species, directly related to the conservation of mass equation of the species (see, e.g., [1], [8]):

$$
\frac{\partial C_{i}}{\partial t}+\nabla N_{i}=\omega_{i}
$$

Here $\omega_{i}$ is the rate of production of the $i$ th species given by the Arrhenius law; it depends on the temperature and is a polynomial function of the $C_{i}$. Conservation of mass requires that

$$
\sum_{i=1}^{S} \frac{\omega_{i}}{M_{i}}=0
$$

We will also assume that

$$
\left|\omega_{i}\left(T, C_{1}, \ldots, C_{s}\right)\right| \leq \kappa_{1}+\kappa_{2}\left(C_{1}+\cdots+C_{s}\right),
$$

although this simplifying assumption is not essential.

\footnotetext{
${ }^{1}$ See, however, many relevant results in [4].
} 
Finally the $N_{i}$ are related to the $C_{j}$ and $\nabla C_{j}$ by the following relations, sometimes called the Stefan-Maxwell equations:

$$
\begin{aligned}
d_{i} N_{i} & +\frac{\mathcal{R} T}{p} \sum_{\substack{j=1 \\
j \neq i}}^{S} d_{i j}\left(C_{j} N_{i}-C_{i} N_{j}\right) \\
& =-\nabla C_{i}-d_{i} C_{i} g \nabla p, \quad i=1, \ldots, S .
\end{aligned}
$$

Here $d_{i}, d_{i j}$ are positive coefficients, $\mathcal{R}$ is the perfect gas constant, and $g$ is a given constant.

The system (2.5) will be analyzed when considered as a linear system for the $N_{i}$ (giving the $N_{i}$ in terms of the $\nabla C_{j}$, the $C_{j}$, and the other functions, $\left.p, T\right)$.

We supplement these equations with one of the following boundary conditions:

Space periodicity: $\Omega$ is the 2 or $3 \mathrm{D}$ rectangle

$\left(0, L_{1}\right) \times\left(0, L_{2}\right)$ or $\left(0, L_{1}\right) \times\left(0, L_{2}\right) \times\left(0, L_{3}\right)$,

and each $C_{i} i=1, \ldots, S$ is periodic (with period $L_{j}$

in the direction $\left.x_{j}, j=1,2,3\right)$.

No flux boundary condition

$$
\frac{\partial C_{i}}{\partial \mathbf{n}}=0 \text { on } \Gamma=\partial \Omega, i=1, \ldots, S
$$

where $\mathbf{n}$ is the unit outward normal on $\Gamma$.

$$
\begin{aligned}
& \text { Dirichlet boundary condition, } \\
& C_{i}=C_{i b} \text { on } \partial \Omega, i=1, \ldots, S,
\end{aligned}
$$

where each $C_{i b}$ is a regular function prescribed and $>0$ on $\partial \Omega$, and arbitrarily extended inside $\Omega$ as a regular positive function.

Our aim in this article is to discuss the spatial discretization of the initial and boundary value problem consisting of $(2.2),(2.5),(2.6)$ and the initial conditions

$$
C_{i}(x, 0)=C_{i b}(x), x \in \Omega, i=1, \ldots, S .
$$

3. Formal a priori estimates. Assuming that all $C_{i}$ 's are positive everywhere and that all functions $C_{i}, N_{i}$ are sufficiently regular, we derive some a priori estimates for the solution $C_{i}$ of (2.2)-(2.6). In Sections 3 and 4, discretized versions of these estimates will be used to establish the stability of the discretized equations. We treat the three boundary conditions $(2.6 \mathrm{a}, \mathrm{b}, \mathrm{c})$, which necessitate some slight changes in the proofs. Finally at the end of this section we show how to guarantee the positivity of the $C_{i}$.

3.1. The linear system (2.5). Equations (2.5) are to be considered as an algebraic linear system ( $S$ equations, $S$ unknowns), which determine at each point $x, t$ the fluxes $N_{i}=N_{i}(x, t)$ in terms of the other quantities in (2.5). 
We rewrite (2.5) in the form

$$
\begin{gathered}
d_{i} C_{i} \frac{N_{i}}{C_{i}}+\frac{\mathcal{R} T}{p} \sum_{\substack{j=1 \\
j \neq i}}^{S} d_{i j} C_{i} C_{j}\left(\frac{N_{i}}{C_{i}}-\frac{N_{j}}{C_{j}}\right) \\
=-\nabla C_{i}-d_{i} C_{i} g \nabla p
\end{gathered}
$$

equivalent to the linear system

$$
\tilde{\mathcal{B}} \tilde{\mathcal{N}}=\mathcal{G}
$$

where $\tilde{\mathcal{N}}=\left(\tilde{N}_{1}, \ldots, \tilde{N}_{S}\right), \tilde{N}_{i}=N_{i} / C_{i}, \mathcal{G}=\left(G_{1}, \ldots, G_{S}\right), G_{i}=-\nabla C_{i}-d_{i} C_{i} g \nabla \rho$, and the matrix $\widetilde{\mathcal{B}}$ reads

$$
\begin{aligned}
& \tilde{\mathcal{B}}_{i j}=-\frac{R T}{p} d_{i j} C_{i} C_{j}, \text { for } i \neq j, \\
& \widetilde{\mathcal{B}}_{i i}=d_{i} C_{i}+\frac{R T}{p} \sum_{\substack{j=1 \\
j \neq i}}^{S} d_{i j} C_{i} C_{j} .
\end{aligned}
$$

The matrix $\widetilde{\mathcal{B}}$ is symmetric positive definite; the positivity follows immediately from the Gershgorin theorem. Hence, solving (3.1), we obtain

The $N_{i}$ are linear functions of $\nabla p$ and the

$\nabla C_{j}$, the coefficients being rational functions of

$p, T$, and all the $C_{k}$ (and the coefficients $\left.d_{i}, R, d_{i j}\right)$.

Let us make further comments depending on the boundary conditions:

- In the periodic case (2.6a), we naturally assume that

$$
p, T \text { are periodic functions (with period } L_{j} \text { in direction } x_{j} \text { ). }
$$

Then, since $C_{j}$ and $\nabla C_{j}$ are assumed to be periodic by (2.6a), the resolution of the system (2.5) or (3.1) shows that

The $N_{i}$ are periodic with period $L_{j}$ in direction $x_{j}, j=1,2,3$,

$$
i=1, \ldots, S \text {. }
$$

- In the case of the no-flux (Neumann) boundary condition (2.6b), we naturally assume that

$$
\frac{\partial p}{\partial \mathbf{n}}=0 \text { on } \Gamma
$$

Then multiplying equations (3.1) by $\mathbf{n}$, for $x \in \Gamma$, we find that

$$
G_{i} \cdot \mathbf{n}=-\frac{\partial C_{i}}{\partial \mathbf{n}}-d_{i} g C_{i} \frac{\partial p}{\partial \mathbf{n}}=0
$$

so that

$$
N_{i} \cdot \mathbf{n}=0 \text { on } \Gamma, i=1, \ldots, S .
$$

We have no particular remark concerning this system in the case (2.6c) (Dirichlet boundary condition). 
3.2. A priori estimates. Assuming that the $C_{i}$ are everywhere positive and smooth, a priori estimates are obtained by multiplying Eq. (2.2) by $\log C_{i}$, integrating over $\Omega$, and adding these equations for $i=1, \ldots, S$. We observe that

$$
\log C_{i} \frac{\partial C_{i}}{\partial t}=\frac{\partial}{\partial t}\left(C_{i} \log C_{i}-C_{i}+2\right)
$$

the constant 2 has been chosen so that the expression between parentheses is bounded from below by a positive number (namely 1). Also

$$
\int_{\Omega} \nabla N_{i} \log C_{i} d x=\int_{\partial \Omega} N_{i} \cdot \mathbf{n} \log C_{i} d x-\int_{\Omega} N_{i} \frac{\nabla C_{i}}{C_{i}} d x .
$$

The integral over $\partial \Omega$ vanishes for both boundary conditions (2.6a) and (2.6b): for (2.6a) by periodicity, for $(2.6 \mathrm{~b})$ because of $(3.7)$; the Dirichlet boundary conditions $(2.6 \mathrm{c})$ will be examined later. There remains, after using (3.1) and summing in $i$ :

$$
\begin{aligned}
\sum_{i=1}^{S} & \int_{\Omega} d_{i} \frac{N_{i}^{2}}{C_{i}} d x+\sum_{\substack{i, j=1 \\
i \neq j}}^{S} \int_{\Omega} \frac{\mathcal{R} T}{p} d_{i j} C_{j} C_{j}\left(\frac{N_{i}}{C_{i}}-\frac{N_{j}}{C_{j}}\right) \frac{N_{i}}{C_{i}} d x \\
& +\sum_{i=1}^{S} \int_{\Omega} d_{i} N_{i} g \nabla p d x \\
= & \sum_{i=1}^{S} \int_{\Omega} d_{i} \frac{N_{i}^{2}}{C_{i}} d x+\sum_{\substack{i, j=1 \\
i \neq j}}^{S} \int_{\Omega} \frac{\mathcal{R} T}{2 p} d_{i j} C_{i} C_{j}\left(\frac{N_{i}}{C_{i}}-\frac{N_{j}}{C_{j}}\right)^{2} d x \\
& +\sum_{i=1}^{S} \int_{\Omega} d_{i} N_{i} g \nabla p d x \\
\geq & \frac{1}{2} \sum_{i=1}^{S} \int_{\Omega} d_{i} \frac{N_{i}^{2}}{C_{i}} d x+\sum_{\substack{i, j=1 \\
i \neq j}}^{S} \int_{\Omega} \frac{\mathcal{R} T}{2 p} d_{i j} C_{i} C_{j}\left(\frac{N_{i}}{C_{i}}-\frac{N_{j}}{C_{j}}\right)^{2} d x \\
& -\frac{1}{2} \sum_{i=1}^{S} \int_{\Omega} C_{i} d_{i}|g \nabla p|^{2} d x .
\end{aligned}
$$

Finally

$$
\begin{aligned}
& \frac{d}{d t} \sum_{i=1}^{S} \int_{\Omega}\left(C_{i} \log C_{i}-C_{i}+2\right) \\
& \quad+\frac{1}{2} \sum_{i=1}^{S} \int_{\Omega} d_{i} \frac{N_{i}^{2}}{C_{i}} d x \\
& \quad+\frac{1}{2} \sum_{\substack{i, j=1 \\
i \neq j}}^{S} \int_{\Omega} \frac{\mathcal{R} T}{2 p} d_{i j} C_{i} C_{j}\left(\frac{N_{i}}{C_{i}}-\frac{N_{j}}{C_{j}}\right)^{2} d x \\
& \leq \frac{1}{2} \sum_{i=1}^{S} \int_{\Omega} C_{i} d_{i}|g \nabla p|^{2} d x+\sum_{i=1}^{S} \int_{\Omega} \omega_{i} \log C_{i} d x .
\end{aligned}
$$


Upon using (2.1) and (2.4), we see that

$$
\frac{d}{d t} \Phi \leq \kappa_{3} \Phi
$$

for some suitable constant $\kappa_{3}$, where

$$
\Phi=\Phi\left(C_{1}, \ldots, C_{s}\right)=\sum_{i=1}^{S} \int_{\Omega}\left(C_{i} \log C_{i}-C_{i}+2\right) d x .
$$

From (3.11) follows the a priori estimate

$$
\Phi(t) \leq \Phi(0) e^{\kappa_{3} T},
$$

valid for $0 \leq t \leq T$. Further estimates can be derived from (3.9), but they will not be used here.

3.3. Dirichlet boundary condition. A similar result is valid in the case of the Dirichlet boundary condition, but the proof must be slightly modified. Instead of multiplying (2.2) by $\log C_{i}$, we multiply this equation by $\log \left(C_{i} / C_{i b}\right)$. The first term gives

$$
\begin{aligned}
\int_{\Omega} & \frac{\partial C_{i}}{\partial t}\left(\log C_{i}-\log C_{i b}\right) \\
= & \frac{d}{d t} \int_{\Omega}\left(C_{i} \log C_{i}-C_{i}+P-C_{i} \log C_{i b}\right) d x \\
& \quad+\int_{\Omega} \frac{C_{i}}{C_{i b}} \frac{\partial C_{i b}}{\partial t} d x
\end{aligned}
$$

Denoting by $\bar{C}_{i b}$ the maximum of $C_{i b}(x \in \Omega, t \in[0, T])$, we now choose the constant $P$ so that

$$
C \log C-C+P-C \log \bar{C}_{i b} \geq 0, \quad \forall C \geq 0 .
$$

The term $\nabla N_{i}$ now yields:

$$
\begin{aligned}
& \int_{\Omega} \nabla N_{i} \log \frac{C_{i}}{C_{i b}} d x \\
& \quad=\int_{\partial \Omega} N_{i} \cdot \mathbf{n} \log \frac{C_{i}}{C_{i b}} d x-\int_{\Omega} N_{i}\left(\frac{\nabla C_{i}}{C_{i}}-\frac{\nabla C_{i b}}{C_{i b}}\right) d x .
\end{aligned}
$$

The term on $\partial \Omega$ vanishes. We then proceed as before and we obtain

$$
\begin{aligned}
\sum_{i=1}^{S} & \int_{\Omega} d_{i} \frac{N_{i}^{2}}{C_{i}} d x+\sum_{\substack{i, j=1 \\
i \neq j}}^{S} \int_{\Omega} \frac{\mathcal{R} T}{\rho} d_{i j} C_{i} C_{j}\left(\frac{N_{i}}{C_{i}}-\frac{N_{j}}{C_{j}}\right) \frac{N_{i}}{C_{i}} d x \\
& +\sum_{i=1}^{S} \int_{\Omega} N_{i}\left(d_{i} g \nabla p+\frac{\nabla C_{i b}}{C_{i b}}\right) d x \\
\geq & \frac{1}{2} \sum_{i=1}^{S} \int_{\Omega} d_{i} \frac{N i^{2}}{C_{i}} d x+\sum_{\substack{i, j=1 \\
i \neq j}}^{S} \int_{\Omega} \frac{\mathcal{R} T}{p} d_{i j} C_{i} C_{j}\left(\frac{N_{i}}{C_{i}}-\frac{N_{j}}{C_{j}}\right)^{2} d x \\
& -\frac{1}{2} \sum_{i=1}^{S} \int_{\Omega} C_{i} d_{i}\left(g \nabla p+\frac{\nabla C_{i b}}{d_{i} C_{i b}}\right)^{2} d x .
\end{aligned}
$$


We then conclude essentially as in (3.11)-(3.12), the term $g \nabla p$ being replaced by $g \nabla p+\nabla C_{i b} / d_{i} C_{i b}$.

3.4. Positivity of the $C_{i}$. In order to guarantee the positivity of the $C_{i}$, we will replace $C_{i}$ by $K_{i}=\log C_{i}$, so that $C_{i}=e^{K_{i}}>0$. Whereas this would have very significant implications to the mathematical theory of these equations (existence, uniqueness issues), we will see that, at the level of the space discretized equations, this produces few changes and we automatically obtain the positivity of the $C_{i}$.

Equations (2.2) and (2.5) become

$$
\begin{gathered}
\frac{\partial K_{i}}{\partial t}+e^{-K_{i}} \nabla N_{i}=e^{-K_{i}} \omega_{i}, i=1, \ldots, S, \\
d_{i} N_{i}+\frac{\mathcal{R} T}{p} \sum_{\substack{j=1 \\
j \neq i}}^{S} d_{i j} e^{K_{i}+K_{j}}\left(e^{-K_{i}} N_{i}-e^{-K_{j}} N_{j}\right) \\
=-e^{K_{i}} \nabla K_{i}-d_{i} e^{K_{i}} g \nabla p, i=1, \ldots, S .
\end{gathered}
$$

The boundary and initial conditions (2.6)-(2.7) are easily transferred to the $K_{i}$.

To obtain the desired a priori estimates, we mimic what we did in Sections 3.2 and 3.3. For the periodic case and the Neumann boundary conditions, we multiply (3.17) by $e^{K_{i}} K_{i}$, integrate over $\Omega$, and add for $i=1, \ldots, S$. With very similar calculations we arrive at (3.9)-(3.12) where, e.g., $\Phi$ is expressed in terms of the $K_{j}$ :

$$
\Phi=\sum_{i=1}^{S} \int_{\Omega}\left(K_{i} e^{K_{i}}-e^{K_{i}}+2\right) d x .
$$

Hence, as in (3.12),

$$
\Phi(t) \leq \Phi(0) e^{\kappa_{3} T}, 0 \leq t \leq T .
$$

In the case of the Dirichlet boundary condition, we multiply $(3.17)$ by $e^{K_{i}}\left(K_{i}-K_{i b}\right)$, $K_{i b}=\log C_{i b}$. The calculations are the same and we obtain again (3.16); as in Sec. 3.3, the term $g \nabla p$ is replaced at the end by $g \nabla p+\nabla C_{i b} / d_{i} C_{i b}=g \nabla p+d_{i}^{-1} \nabla K_{i b}$.

4. Spatial discretization by finite differences. We now discuss the spatial discretization of the above equations using finite differences (Sec. 4) or pseudo-spectral methods (Sec. 5). The three boundary conditions $(2.6 \mathrm{a}, \mathrm{b}, \mathrm{c})$ will be successively considered but, for the sake of simplicity, we will assume in all cases that $\Omega$ is a $2 \mathrm{D}$ or $3 \mathrm{D}$ rectangle, $\Omega=\left(0, L_{1}\right) \times\left(0, L_{2}\right)$ or $\Omega=\left(0, L_{1}\right) \times\left(0, L_{2}\right) \times\left(0, L_{3}\right)$. The one-dimensional case, $\Omega=\left(0, L_{1}\right)$, can be considered as well.

4.1. Space periodic case. In the case of finite differences with meshes $h_{i}=\Delta x_{i}=$ $L_{i} / R_{i}, i=1,2,3$, we consider the nodal points $r h=\left(r_{1} h_{1}, r_{2} h_{2}, r_{3} h_{3}\right), h=\left(h_{1}, h_{2}, h_{3}\right)$, $r=\left(r_{1}, r_{2}, r_{3}\right),-1 \leq r_{i} \leq R_{i}+1$. We discretize, for instance, (2.2) with a forward discretization scheme corresponding to $\nabla_{h}$ with, e.g.,

$$
\nabla_{1 h} \varphi(x)=\frac{\varphi\left(x_{1}+h_{1}, x_{2}, x_{3}\right)-\varphi\left(x_{1}, x_{2}, x_{3}\right)}{h_{1}} .
$$

Hence

$$
\frac{d C_{i h}}{d t}+\nabla_{h} N_{i h}=\omega_{i}
$$


equations (4.1) being understood for all times, and at points $r h, 0 \leq r_{i} \leq R_{i}$, forming the mesh $\Omega_{R}$. Space periodicity is enforced by imposing that

$$
\left.C_{i h}\right|_{x_{j}=0}=\left.C_{i h}\right|_{x_{j}=R_{j} h_{j}=L_{j}}, \quad j=1,2,3, i=1, \ldots, S .
$$

Also, we extend the value of $C_{i}$ at points $-h_{j}$ and $\left(R_{j}+1\right) h_{j}$, by periodicity:

$$
\left.C_{i h}\right|_{x_{j}=-h_{j}}=\left.C_{i h}\right|_{x_{j}=\left(R_{j}-1\right) h_{j}},\left.C_{i h}\right|_{x_{j}=\left(R_{j}+1\right) h_{j}}=\left.C_{i h}\right|_{x_{j}=h_{j}} .
$$

We then define the backward finite difference operator $\bar{\nabla}_{h}=\left(\bar{\nabla}_{1 h}, \bar{\nabla}_{2 h}, \bar{\nabla}_{3 h}\right)$ with, e.g.,

$$
\bar{\nabla}_{1 h} \varphi(x)=\frac{\varphi\left(x_{1}, x_{2}, x_{3}\right)-\varphi\left(x_{1}-h_{1}, x_{2}, x_{3}\right)}{h_{1}} .
$$

Before discretizing Eq. (2.5), we rewrite it in the following equivalent form:

$$
\begin{aligned}
d_{i} \frac{N_{i}}{C_{i}} & +\frac{\mathcal{R} T}{p} \sum_{\substack{j=1 \\
j \neq i}}^{S} d_{i j} C_{j}\left(\frac{N_{i}}{C_{i}}-\frac{N_{j}}{C_{j}}\right) \\
& =-\frac{\nabla C_{i}}{C_{i}}-d_{i} g \nabla p, \quad i=1, \ldots, n .
\end{aligned}
$$

The discretized form of (2.5)-(4.4) then reads

$$
\begin{aligned}
d_{i} \frac{N_{i h}}{C_{i h}} & +\frac{\mathcal{R} T}{p} \sum_{\substack{j=1 \\
j \neq i}}^{S} C_{j h}\left(\frac{N_{i h}}{C_{i h}}-\frac{N_{j h}}{C_{j h}}\right) \\
& =-\bar{\nabla}_{h} \log C_{i h}-d_{i} g \nabla p, \quad i=1, \ldots, n,
\end{aligned}
$$

these equations being written at all points of $\Omega_{h}$ (using (4.2) and (4.3)).

Note that the equations (4.1) and (4.5) written at the points of $\Omega_{h}$ are not all independent. Because of periodicity they are valid at all these points, but the independent equations are obtained, e.g., at the points of $\Omega_{h}^{-}$consisting of the points $r h, 0 \leq r_{j} \leq R_{j}-1$, $i=1,2,3$.

To avoid the difficulties with the sign of $C_{i h}$, we introduce as in Sec. 3.4 the functions $K_{i h}=\log C_{i h}$ and express (4.1) and (4.5) in terms of the $K_{i h}$. The discrete analogues of (3.12) provide a priori estimates that promptly imply the existence of the $K_{i h}$ for all time and that guarantee stability of the schemes.

The discrete analogue of $\Phi$ is now

$$
\Phi_{h}=\Phi_{h}(t)=\sum_{x \in \Omega_{h}^{-}} \sum_{i=1}^{S}\left(K_{i}(x, t) e^{K_{i}(x, t)}-e^{K_{i}(x, t)}+2\right) .
$$

We multiply (4.1) written at $x \in \Omega_{h}^{-}$, by $K_{i h}(x, t)$, and add the equations for $x \in \Omega_{h}^{-}$, $i=1, \ldots, S$. We proceed as in the continuous case, using the discrete integration by parts relation

$$
\sum_{x \in \Omega_{h}^{-}} \nabla_{h} N_{i h}(x) K_{i h}(x)=-\sum_{x \in \Omega_{h}^{-}} N_{i h}(x) \cdot \bar{\nabla}_{h} K_{i h}(x),
$$

which is valid here because the functions $N_{i h}$ and $K_{i b}$ are periodic in the sense of (4.2) and (4.3). To check (4.6) we fix $i, 1 \leq i \leq S, j, 1 \leq j \leq 3$ (say $j=1$ ), $x_{2}=r_{2} h_{2}$, and 
$x_{3}=r_{3} h_{3}, 1 \leq r_{2} \leq R_{2}-1,1 \leq r_{3} \leq R_{3}-1$. We then call $V_{s}$ the value of $N_{i h}$ at $s h_{1}$, and $w_{s}$ the value of $K_{i h}$ at the same point. After multiplication by $h_{1}$, the corresponding contributions to the left- and right-hand sides of (4.6) are respectively

$$
\begin{aligned}
& \left(V_{1}-V_{0}\right) W_{0}+\cdots+\left(V_{R_{1}}-V_{R_{1}-1}\right) W_{R_{1}-1} \\
& =-V_{0} W_{0}-V_{1}\left(W_{1}-W_{0}\right)-\cdots-V_{R_{1}-1}\left(W_{R_{1}-1}-W_{R_{1}-2}\right)+V_{R_{1}} W_{R_{1}-1},
\end{aligned}
$$

and

$$
-V_{0}\left(W_{0}-W_{-1}\right)-V_{1}\left(W_{1}-W_{0}\right)-\cdots-V_{R_{1}-1}\left(W_{R_{1}-1}-W_{R_{1}-2}\right) ;
$$

these two sums are the same since $V_{0}=V_{R_{1}}$ and $W_{-1}=W_{R_{1}-1}$. Hence (4.6).

4.2. No-flux (Neumann) boundary condition. For the no-flux (Neumann) boundary condition the mesh $\Omega_{h}$ is the same as before, corresponding to the points $r h=$ $\left(r_{1} h_{1}, r_{2} h_{2}, r_{3} h_{3}\right), 0 \leq r_{j} \leq R_{j}, h_{j}=L_{j} / R_{j}, j=1,2,3$. The interior mesh $\Omega_{h}^{-}$consists now of the points $r h$, for $1 \leq r_{j} \leq R_{j}-1, j=1,2,3$. The independent (unknown) values of the $C_{i h}$ or $K_{i h}=\log \left(C_{i h}\right)$, are the values of the $C_{i h}$ at the points of $\Omega_{h}^{-}$. The boundary condition $(2.6 \mathrm{~b})$ is enforced by setting

$$
\begin{gathered}
\left.C_{i h}\right|_{x_{j}=0}=\left.C_{i h}\right|_{x_{j}=h_{j}},\left.C_{i h}\right|_{x_{j=\left(R_{j}-1\right) h_{j}}}=\left.C_{i h}\right|_{x_{j}=R_{j} h_{j}=L_{j}}, \\
j=1,2,3, i=1, \ldots, S
\end{gathered}
$$

we thus extend the definition of the $C_{i h}$ to all the points of $\Omega_{h}^{-}$. For consistency, and in view of (3.7), we discretize $p$ by setting $p_{h}(x)=p(x)$, for $x \in \Omega_{h}^{-}$and we set, as in (4.8),

$$
\left.p_{h}\right|_{x_{j}=0}=\left.p_{h}\right|_{x_{j}=h_{j}},\left.p_{h}\right|_{x_{j}=\left(R_{j}-1\right) h_{j}}=\left.p_{h}\right|_{x_{j}=R_{j} h_{j}=L_{j}},
$$

thus introducing an $0(h)$ error in the discretization of $p$.

The discretized equations (4.5) are written at all points $x \in \Omega_{h}^{+}$of the form $r h=$ $\left(r_{1} h_{1}, r_{2} h_{2}, r_{3} h_{3}\right), 1 \leq r_{j} \leq R_{j}, j=1,2,3$. Thus the $N_{i h}$ are defined at all points $x \in \Omega_{h}^{+}$. In view of (4.8), (4.9), and the discussion before (3.8),

$$
\begin{aligned}
& \text { The } j^{\text {th }} \text { component of } N_{i h} \text { vanishes at } x_{j}=h_{j} \\
& \text { and } x_{j}=R_{j} h_{j}=L_{j}, j=1,2,3, i=1, \ldots, S \text {. }
\end{aligned}
$$

Setting $C_{i h}=\log K_{i h}$, we write the equation (4.1) for the $K_{i h}$ at all points $x \in \Omega_{h}$. The resolution on some interval of time $\left(0, t_{h}\right)$ of the system (4.1) supplemented by (4.5) and the initial conditions $(2.7)$ for the $C_{i h}\left(K_{i h}\right)$ at $x \in \Omega_{h}^{-}$is standard. The discrete analog of the estimate (3.17) is valid; it guarantees that the $K_{i h}$ are defined on the whole interval $[0, T]$, and thus the $C_{i h}=\exp \left(K_{i h}\right)$ are defined and positive on the whole interval $[0, T]$. Furthermore, the discrete analog of (3.17) provides the stability of the space discretized equations. In the course of proving this estimate, we make use of (4.6), which is still valid. With the same notation as for (4.7), the proof of (4.6) ends up in showing that the following sums are the same:

$$
\begin{gathered}
\left(V_{2}-V_{1}\right) W_{1}+\cdots+\left(V_{R_{1}}-V_{R_{1}-1}\right) W_{R_{1}-1} \\
=-V_{1} W_{1}-V_{2}\left(W_{2}-W_{1}\right) \cdots-V_{R_{1}-1}\left(W_{R_{1}-1}-W_{R_{1}-2}\right) \\
+V_{R_{1}} W_{R_{1}-1},
\end{gathered}
$$

and

$$
-V_{1}\left(W_{1}-W_{0}\right) \cdots-V_{R_{1}-1}\left(W_{R_{1}-1}-W_{R_{1}-2}\right)
$$


These sums are indeed the same since by (4.10) (and the notation in (4.7)), $V_{1}=V_{R_{1}}=0$.

The proof is now complete for the Neumann boundary condition case.

4.3. Dirichlet boundary condition. In this case the notation for the meshes is as in Sec. 4.2: $\Omega_{h}$ is the set of points $r h, 0 \leq r_{j} \leq R_{j}, j=1,2,3$, and $\Omega_{h}^{-}$is the set of points $r h, 1 \leq r_{j} \leq R_{j}-1, j=1,2,3$. The independent (unknown) values of the $C_{i h}$ (or $\left.K_{i h}=\log C_{i h}\right)$ are the values at the points of $\Omega_{h}^{-}$. The boundary condition (2.6c) is enforced by setting

$$
\begin{aligned}
& C_{i h}=C_{i b} \text { at } x_{j}=0 \text { or } R_{j}, \\
& j=1,2,3, i=1, \ldots, S
\end{aligned}
$$

we thus extend the definition of the $C_{i h}$ (or $K_{i h}$ ) at all points of $\Omega_{h}$.

Similarly, $K_{i b h}$ (or $C_{i b h}$ ) is defined at all points of $\Omega_{h}$ by just setting

$$
K_{i b h}(x)=K_{i b}(x), x \in \Omega_{h} .
$$

The discretized equations (4.5) are written at all points $x \in \Omega_{h}^{+}$of the form $r h=$ $\left(r_{1} h_{1}, r_{2} h_{2}, r_{3} h_{3}\right), 1 \leq r_{j} \leq R_{j}, j=1,2,3$. Thus the $N_{i h}$ are defined at all points $x \in \Omega_{h}^{+}$.

Setting $C_{i h}=\log K_{i h}$, we write the equations (4.1) for the $K_{i h}$ at all points $x \in \Omega_{h}^{-}$. The resolution on some interval $\left(0, t_{h}\right)$ of this system, supplemented by equations (4.5) and the initial conditions $(2.7)$ for the $C_{i h}\left(K_{i h}\right)$ at $x \in \Omega_{h}^{-}$, is standard.

We now proceed with the discrete analog of the estimate (3.17) to show that $t_{h}=T$, and to establish the stability.

At each $x \in \Omega_{h}^{-}$, Eq. (4.1) is multiplied by $\left(K_{i h}-K_{i b h}\right)(x)$; we then sum for $x \in \Omega_{h}^{-}$ and $i=1, \ldots, S$. The main difficulty is in establishing the discrete integration by parts formula (4.6) which is now replaced by

$$
\begin{aligned}
& \sum_{x \in \Omega_{h}^{-}} \nabla_{h} N_{i h}(x)\left(K_{i h}-K_{i b h}\right)(x) \\
& =-\sum_{x \in \Omega_{h}^{+}} N_{i h}(x) \bar{\nabla}_{h}\left(K_{i h}-K_{i b h}\right)(x) .
\end{aligned}
$$

To prove (4.14) we proceed as in (4.7) with a similar notation: $V_{j}$ is still the value of (say) the first component of $N_{i h}\left(x_{1}, x_{2}, x_{3}\right)$ at $x_{1}=j h_{1}$, and $W_{j}$ is the value at the same point of $\left(K_{i h}-K_{i b h}\right)\left(x_{1}, x_{2}, x_{3}\right), x_{1}=j h_{1}$. The sums to be compared are now

$$
\begin{aligned}
& \left(V_{2}-V_{1}\right) W_{1}+\left(V_{3}-V_{2}\right) W_{2}+\cdots+\left(V_{R_{1}}-V_{R_{1}-1}\right) W_{R_{1}-1}, \\
& \text { and } \\
& \quad-V_{1}\left(W_{1}-W_{0}\right) \cdots-V_{R_{1}}\left(W_{R_{1}}-W_{R_{1}-1}\right) .
\end{aligned}
$$

We add $\left(V_{R_{1}+1}-V_{R_{1}}\right) W_{R_{1}}$ to the first sum, this term vanishing $\left(W_{R_{1}}=0 ; V_{R_{1}+1}\right.$ arbitrary). Remembering that $W_{0}$ vanishes like $W_{R_{1}}$, we conclude that the two sums are the same, and (4.14) follows.

The stability is proven in the Dirichlet case as well. 
5. Spatial discretization by pseudo-spectral methods. In this section we discuss the spatial discretization of the boundary value problem (2.2)-(2.7) using pseudospectral methods. We will use trigonometric polynomials based on equidistant points in the space-periodic case and Legendre polynomials based on the Gauss-Lobato points for the Neumann and Dirichlet boundary conditions. The domain $\Omega$ is a $2 \mathrm{D}$ rectangle, $\Omega=\left(0, L_{1}\right) \times\left(0, L_{2}\right)$, or a $3 \mathrm{D}$ parallelepiped, $\Omega=\left(0, L_{1}\right) \times\left(0, L_{2}\right) \times\left(0, L_{3}\right)$. The (simpler) one-dimensional case, $\Omega=\left(0, L_{1}\right)$, can be treated in the same manner.

5.1. Space periodic case. In each direction $O x_{k}, k=1,2,3$, we consider the equidistant points

$$
x_{k}^{i}=\frac{i L_{k}}{R_{k}}, i=0, \ldots, 2 R_{k}-1 .
$$

We denote by $\Omega_{R}$ the set of points $Y=\left(x_{1}^{i_{2}}, x_{2}^{i_{2}}, x_{3}^{i_{3}}\right), 0 \leq i_{1} \leq 2 R_{1}-1,0 \leq i_{2} \leq 2 R_{2}-1$, $0 \leq i_{3} \leq 2 R_{3}-1$, and by $\Omega_{R}^{-}$the set of points $x=\left(x_{1}^{i_{1}}, x_{2}^{i_{2}}, x_{3}^{i_{3}}\right), 0 \leq i_{1} \leq 2 R_{1}-1$, $0 \leq i_{2} \leq 2 R_{2}-1,0 \leq i_{3} \leq 2 R_{3}-1$.

Each function is determined by its values at the points of $\Omega_{R}^{-}$; the nodal values at the points of $\Omega_{R} \backslash \Omega_{R}^{-}$are determined by periodicity, thus enforcing the boundary condition (2.6a). The function is then extended to all of $\Omega$ (and to the whole space $\mathbb{R}^{3}$ or $\mathbb{R}^{2}$ ) by trigonometric polynomial interpolation in each direction. For instance, the interpolation in the direction $O x_{1}$ of a function $f$ reads

$$
I_{R_{1}} f\left(x_{1}, x_{2}, x_{3}\right)=\sum_{j=0}^{2 R_{1}-1} f\left(x_{1}^{j}, x_{2}, x_{3}\right) g_{j}\left(x_{1}\right)
$$

where $x_{2}$ and $x_{3}$ are fixed (of the form (5.1)). The Lagrange polynomials $g_{j}$ belong to the space $\widetilde{\mathcal{P}}_{R_{1}}$ (this is the space spanned by the polynomials $\cos \left(m \pi x_{1}\right), 0 \leq m \leq R_{1}$, and $\left.\sin \left(m \pi x_{1}\right), 1 \leq m \leq R_{1}-1\right)$ and satisfy

$$
g_{j}\left(x_{1}^{k}\right)=\delta_{j k}, 0 \leq j, k \leq 2 R_{1}-1 .
$$

Before we continue, let us recall also that the (exact) derivative of $I_{R_{1}} f$ in the direction $O x_{1}$ belongs also to $\widetilde{\mathcal{P}}_{R_{1}}^{1}$ and it is characterized by its nodal values:

$$
\frac{\partial}{\partial x_{1}} I_{R_{1}} f\left(x_{1}^{k}, x_{2}, x_{3}\right)=\sum_{j=0}^{2 R_{1}-1} f\left(x_{1}^{j}, x_{2}, x_{3}\right) \frac{d g_{j}}{d x_{1}}\left(x_{1}^{k}\right), k=0, \ldots, 2 R_{1}-1 .
$$

$\left\{\frac{d g_{j}}{d x_{1}}\left(x_{1}^{k}\right)\right\}_{0 \leq k \leq 2 R_{1}-1}$ is determined from the vector $\left\{g_{j}\left(x_{1}^{k}\right)\right\}_{0 \leq k \leq 2 R_{1}-1}$ by a linear transformation (matrix-vector product), and the differentiation matrix can be built by Fast Fourier Transform or other suitable fast methods.

The Fourier method involves seeking a trigonometrical polynomial (in all three space directions) $K_{i}\left(x_{1}, x_{2}, x_{3}, t\right)$ such that equation (4.4),

$$
\begin{aligned}
d_{i} \frac{N_{i}}{C_{i}} & +\frac{\mathcal{R} T}{p} \sum_{\substack{j=1 \\
j \neq i}}^{S} d_{i j} C_{j}\left(\frac{N_{i}}{C_{i}}-\frac{N_{j}}{C_{j}}\right) \\
& =-\frac{\nabla C_{i}}{C_{i}}-d_{i} g \nabla p, i=1, \ldots, n
\end{aligned}
$$


is valid in $\Omega$, and

$$
\begin{aligned}
& \frac{\partial K_{i}(x, t)}{\partial t}+e^{-K_{i}(x, t)} \nabla I_{R} N_{i}(x, t) \\
& \quad=e^{-K_{i}(x, t)} \omega_{i}(x, t), i=1, \ldots, S, 0<t<T_{*} .
\end{aligned}
$$

In the following we will establish the stability of the Fourier discretization of (3.1). We comment here that we use the notation $K_{i}$ for the Fourier approximation instead of the more correct one $K_{i}^{N}$; this is done for simplicity.

We multiply $(5.5)$ by $e^{K_{i}(x, t)} K_{i}(x, t) \varpi, \varpi=\Pi_{k=1}^{3}\left(\frac{L_{k}}{2 R_{k}}\right)$, and sum for $x \in \Omega_{R}^{-}$. We obtain a discrete integration formula which is exact for the functions interpolated by $I_{R}$, because the product $\left(I_{R} K_{i}\right)\left(\nabla I_{R} N_{i}\right)$ belongs to $\mathcal{P}_{2 R_{k}-1}^{k}$ in each direction $k, \mathcal{P}_{2 R-1}$ being the space of trigonometric polynomials of degree $\leq 2 R-1$ (in one direction). It is then legitimate to integrate by parts, i.e., using periodicity:

$$
\int_{\Omega}\left(K_{i}\right)\left(\nabla I_{R} N_{i}\right)(x, t) d x=-\int_{\Omega}\left(\nabla K_{i}\right)\left(I_{R} N_{i}\right)(x, t) d x .
$$

For the same reason as before, the integral in the right-hand side of (5.6) is exactly equal to the (opposite of the) sum of the $\left(\nabla K_{i}(x, t)\right) N_{i}(x, t) \varpi$ for the $x$ in $\Omega_{R}^{-}$. As explained before, $\nabla K_{i}$ at the points $x \in \Omega_{R}^{-}$is given by the discrete version of equations (4.4). The analysis continues without any further change until we obtain the discrete analog of (3.18) and (3.19) for

$$
\Phi_{R}=\Phi_{R}(t)=\sum_{x \in \Omega_{R}^{-}}\left(K_{i}(x, t) e^{K_{i}(x, t)}-e^{K_{i}(x, t)}+2\right) \varpi .
$$

5.2. No-flux (Neumann) boundary condition. For the no-flux (Neumann) boundary condition (2.6b), the mesh $\Omega_{R}$ consists of the Gauss-Lobato points in each direction $0 x_{k}$, i.e., $x=\left(x_{1}^{i_{1}}, x_{2}^{i_{2}}, x_{3}^{i_{3}}\right), 0 \leq i_{1} \leq R_{1}, 0 \leq i_{2} \leq R_{2}, 0 \leq i_{3} \leq R_{3}$, and for $k=1,2,3$.

The $x_{k}^{i}$ are the roots of $Q(s)=\left(1-s^{2}\right) P_{R_{k}}^{\prime}(s)$,

$P_{R_{k}}$ being the Legendre polynomial of degree $R_{k}, 0 \leq i \leq R_{k}$.

In, say, the direction $0 x_{1}$, the interpolation $I_{R_{1}} f$ of a function $f$ is

$$
I_{R_{1}} f\left(x_{1}, x_{2}, x_{3}\right)=\sum_{j=0}^{R_{1}} f\left(x_{1}^{j}, x_{2}, x_{3}\right) g_{j}\left(x_{1}\right)
$$

and

$$
g_{j}(s)=\frac{Q(s)}{Q^{\prime}\left(x_{1}^{j}\right)\left(s-x_{1}^{j}\right)},
$$

$x_{2}, x_{3}$ being fixed.

We recall that the $g_{j}$ satisfy:

$$
g_{j}\left(x_{1}^{k}\right)=\delta_{j k}, 0 \leq j, k \leq R_{1},
$$

and that they form a basis of the space $\mathcal{P}_{R_{1}}=\mathcal{P}_{R_{1}}(s)$ of algebraic polynomials of degree $\leq R_{1}$ in the (one-dimensional) variable $s$. The space $\mathcal{P}_{R}$ is the space of polynomials in $x_{1}, x_{2}, x_{3}$, spanned by the polynomials in $\mathcal{P}_{R_{1}}\left(x_{1}\right) \cdot \mathcal{P}_{R_{2}}\left(x_{2}\right) \cdot \mathcal{P}_{R_{3}}\left(x_{3}\right)$. 
We recall also that (in dimension 1) the discrete integration formula

$$
\int_{0}^{L_{1}} f(s) d s \simeq \sum_{j=0}^{R_{1}} f\left(x_{1}^{j}\right) \varpi_{1}^{j}\left(x_{1}^{j}\right)
$$

is exact for all polynomials $f$ of degrees $\leq 2 R_{j}-1$, where the $\varpi_{1}^{j_{1}}$ are the weights of the Legendre-Lobato formula. We write also, for $x=\left(x_{1}^{i_{1}}, x_{2}^{i_{2}}, x_{3}^{i_{3}}\right) \in \Omega_{R}$,

$$
\varpi(x)=\varpi_{1}^{i_{1}}\left(x_{1}^{i_{1}}\right) \varpi_{2}^{i_{2}}\left(x_{2}^{i_{2}}\right) \varpi_{3}^{i_{3}}\left(x_{3}^{i_{3}}\right) .
$$

Hence, for a function $f$ defined on $\Omega$, the quadrature formula

$$
\int_{\Omega} f(x) d x \simeq \sum_{x \in \Omega_{R}} f(x) \varpi(x)
$$

is exact for a polynomial $f$ of degree $\leq 2 R_{j}-1$ in each variable $x_{j}, j=1,2,3$.

The exact derivative of $I_{R_{1}} f$ in direction $x_{1}$ belongs, of course, to $\mathcal{P}_{R_{1}}\left(x_{1}\right)\left(\mathcal{P}_{R_{1}-1}\left(x_{1}\right)\right.$ in fact), and it is thus fully characterized by its nodal values:

$$
\frac{\partial}{\partial x_{1}} I_{R_{1}} f\left(x_{1}^{k}, x_{2}, x_{3}\right)=\sum_{j=0}^{R_{1}} f\left(x_{1}^{j}, x_{2}, x_{3}\right) \frac{d g_{j}}{d x_{1}}\left(x_{1}^{k}\right), k=0, \ldots, R_{1} .
$$

The differentiation matrix $\left\{\frac{d g_{j}\left(x_{1}^{k}\right)}{d x_{1}}\right\}_{0 \leq j, k \leq R_{1}}$ can be written explicitly or evaluated by fast transforms.

Let us now proceed with the resolution of our system. We call $\Omega_{R}^{0}$ the set of points $x=\left(x_{1}^{i_{1}}, x_{2}^{i_{2}}, x_{3}^{i_{3}}\right)$, the $x_{k}^{j}$ as in (5.7) and with $1 \leq i_{k} \leq R_{k}-1, k=1,2,3$.

For two functions $f$ and $g$, we have of course the (exact) integration by parts formula

$$
\begin{aligned}
\int_{0}^{L_{1}} & \frac{\partial}{\partial x_{1}} I_{R}\left[f\left(x_{1}\right)\right] \cdot I_{R}\left[g\left(x_{1}\right)\right] d x_{1} \\
= & I_{R}\left[f\left(L_{1}\right)\right] I_{R}\left[g\left(L_{1}\right)\right]-I_{R}[f(0)] I_{R}[g(0)] \\
& -\int_{0}^{L_{1}} I_{R}\left[f\left(x_{1}\right)\right] \cdot \frac{\partial}{\partial x_{1}} I_{R}\left[g\left(x_{1}\right)\right] d x_{1},
\end{aligned}
$$

equivalent to

$$
\begin{aligned}
\sum_{j=0}^{R_{1}} & \frac{\partial}{\partial x_{j}} I_{R}\left[f\left(x_{1}^{j}\right)\right] g\left(x_{1}^{j}\right) \varpi_{1}^{j} \\
= & f\left(x_{1}^{R_{1}}\right) g\left(x_{1}^{R_{1}}\right)-f\left(x_{1}^{0}\right) g\left(x_{1}^{0}\right) \\
& -\sum_{j=0}^{R_{1}} f\left(x_{1}^{j}\right) \frac{\partial}{\partial x_{1}} I_{R}\left[g\left(x_{1}^{j}\right)\right] \varpi_{1}^{j}
\end{aligned}
$$


In the case, useful below, where $f(0)=f\left(L_{1}\right)=0$, these formulas become

$$
\begin{aligned}
& \int_{0}^{L_{1}} \frac{\partial}{\partial x_{1}} I_{R}\left[f\left(x_{1}\right)\right] \cdot I_{R}\left[g\left(x_{1}\right)\right] d x_{1} \\
& =-\int_{0}^{L_{1}} I_{R}\left[f\left(x_{1}\right)\right] \cdot \frac{\partial}{\partial x_{1}} I_{R}\left[g\left(x_{1}\right)\right] d x_{1}, \\
& \sum_{j=0}^{R_{1}} \frac{\partial}{\partial x_{1}} I_{R}\left[f\left(x_{1}^{j}\right)\right] f\left(x_{1}^{j}\right) \varpi_{1}^{j}\left(x_{1}^{j}\right) \\
& \quad=-\sum_{j=0}^{R_{1}} f\left(x_{1}^{j}\right) \frac{\partial}{\partial x_{1}} I_{R}\left[g\left(x_{1}^{j}\right)\right] \varpi_{1}^{j}\left(x_{1}^{j}\right) .
\end{aligned}
$$

Before we proceed with the resolution of our system, we need to comment here on the discrete implementation of the boundary condition $(2.6 \mathrm{~b})$ which will be done by weak (variational) formulation, as is done, in a standard way, with, e.g., finite elements. We start with the continuous (nondiscrete) case and we first make the remark converse to (3.6)-(3.7); namely, if the conditions (3.6) and (3.7) hold, then (3.1) shows that $\mathbf{n} \cdot \nabla C_{i}=\partial C_{i} / \partial \mathbf{n}=0$ on $\Gamma$, for $i=1, \ldots, S$. Consequently when $p$ satisfies (3.6), the conditions (2.6b) and (3.7) are equivalent. Hence we will implement (2.6b) by imposing the conditions (3.7). More precisely, multiplying (2.2) by a test function $\Phi$ and integrating over $\Omega$ gives, with (3.7):

$$
\begin{aligned}
& \int_{\Omega} \frac{\partial C_{i}}{\partial t} \Phi d x+\int_{\Omega} \nabla N_{i} \Phi d x=\int_{\Omega} \omega_{i} \Phi d x \\
& \int_{\Omega} \nabla N_{i} \Phi d x=\int_{\Gamma} N_{i} \mathbf{n} \Phi d \Gamma-\int_{\Omega} N_{i} \cdot \nabla \Phi d x \\
& =-\int_{\Omega} N_{i} \cdot \nabla \Phi d x
\end{aligned}
$$

Thus

$$
\int_{\Omega} \frac{\partial C_{i}}{\partial t} \Phi d x-\int_{\Omega} N_{i} \cdot \nabla \Phi d x=\int_{\Omega} \omega_{i} \Phi d x,
$$

or equivalently with $C_{i}=e^{K_{i}}$,

$$
\int_{\Omega} \frac{\partial}{\partial t} e^{K i} \Phi d x-\int_{\Omega} N_{i} \cdot \nabla \Phi d x=\int_{\Omega} \omega_{i} \Phi d x .
$$

Also (this is a standard exercise in variational formulations) if (5.17) is valid for all smooth test functions $\Phi$, then necessarily $(2.2)$ holds and $N_{i} \cdot \mathbf{n}=0$ on $\Gamma$. Thus we intend now to approximate $(2.2),(2.6 \mathrm{~b})$ by implementing a discrete pseudo-spectral form of $(5.17)^{2}$.

We call $K_{i R}$ an approximation of $K_{i}$ defined at the nodal points of $\Omega_{R}$ and $C_{i R}=e^{K_{i R}}$, and we introduce the interpolation $I_{R} K_{i R}$ as before. We now approximate (5.18) in the

\footnotetext{
${ }^{2}$ We could have done the same for finite differences, but the discretization of the boundary condition was then simpler than in the present case.
} 
following form:

$$
\begin{gathered}
\frac{\partial}{\partial t} \sum_{x \in \Omega_{R}} e^{K_{i R}}(x, t) \Phi(x)-\int_{\Omega} I_{R}\left(N_{i R}\right) \nabla \Phi d x=\int I_{R}\left(\omega_{i}\right) \Phi d x, \\
\forall \Phi \in \mathcal{P}_{R}, i=1, \ldots, S .
\end{gathered}
$$

Here the $N_{i R}$ are defined on $\Omega_{R}$ by the resolution of equations (3.1) written in the form (4.4) at $x \in \Omega_{R} ; x \in \Omega_{R}$ is of the form $x=\left(x_{1}^{i_{1}}, x_{2}^{i_{2}}, x_{3}^{i_{3}}\right), 0 \leq i_{1} \leq R_{1}, 0 \leq i_{2} \leq R_{2}$, $0 \leq i_{3} \leq R_{3}$. Furthermore, $\nabla C_{i} / C_{i}$ is replaced by the value of $\nabla I_{R} K_{i R}$ and $\nabla p$ is replaced by $\nabla I_{R} p_{R}$, where $p_{R}=p$ at the points $x$ of $\Omega_{R}$. As indicated in (5.19), we impose (5.19) to be valid for every $\Phi \in \mathcal{P}_{R}$, for $i=1, \ldots, S$.

For each $i,(5.19)$ is an ordinary differential system which we solve in classical ways by advancing in time.

The existence of the $K_{i R}$ on a finite interval of time $\left(0, T_{*}\right), 0<T_{*} \leq T$ is standard and the a priori estimate similar to (3.18) shows that actually $T_{*}=T$. To derive this a priori estimate, we replace $\Phi$ by $I_{R} K_{i R}$ in (5.19). We use the integration formulas derived from (5.9), taking advantage of the fact that these integration formulas are exact for $I_{R} N_{i R} \nabla K_{i R}$. Using then equations (4.4) at the points $x \in \Omega_{R}$, we conclude, as in the continuous case, that

$$
-\int_{\Omega} I_{R} N_{i R} \nabla K_{i R} d x \geq 0
$$

There remains

$$
\int_{\Omega} \frac{\partial}{\partial t} I_{R}\left(e^{K_{i R}}\right) K_{i R} d x \leq \int_{\Omega}\left(I_{R} \omega_{i}\right) K_{i R} d x,
$$

and we derive the analogue of (3.18) as in the continuous case.

REMARK 5.1. We should be able to improve the approximation of (3.8) and thus to improve the approximation by choosing $p_{R}$ in such a way that

$$
\frac{\partial p_{i R}}{\partial \mathbf{n}}=0 \text { on } \Gamma
$$

To this end we choose $p_{R}(x)=p(x)$ at all points $x$ of $\Omega_{R}^{0}$, the set of points $x=$ $\left(x_{1}^{i_{1}}, x_{2}^{i_{2}}, x_{3}^{i_{3}}\right)$, the $x_{k}^{j}$ as in (5.7) and with $1 \leq i_{k} \leq R_{k}-1, k=1,2,3$. Then we define $p_{R}$ on $\Omega_{R} \backslash \Omega_{R}^{0}$ so that the relations (5.20) hold. For instance, in direction $0 x_{1}$, and for $x_{2}, x_{3}$ fixed, we would define $\left.p_{R}\right|_{x_{1}^{0}}$ and $\left.p_{R}\right|_{x_{1}^{R_{1}}}$ by imposing the following conditions (see (5.8) and (5.12)):

$$
\begin{gathered}
\frac{\partial}{\partial x_{1}} p_{R}\left(x_{1}^{k}, x_{2}, x_{3}\right)=\sum_{j=0}^{R_{1}} p_{R}\left(x_{1}^{k}, x_{2}, x_{3}\right) \frac{d g_{j}}{d x_{1}}\left(x_{1}^{k}\right)=0 \\
\text { for } k=0 \text { and } R_{1} .
\end{gathered}
$$

The unknowns in (5.21) are $p_{R}\left(x_{1}^{0}, x_{2}, x_{3}\right)$ and $p_{R}\left(x_{1}^{R_{1}}, x_{2}, x_{3}\right)$; the unique solvability of (5.21) is classical [7]. 
5.3. Dirichlet boundary condition. The framework is the same as for the Neumann pseudo-spectral case. The unknowns are the values of the $K_{i R}$ on $\Omega_{R}$; of course, on $\Omega_{R} \backslash \Omega_{R}^{0}$, we impose

$$
\begin{gathered}
K_{i R}=K_{i b} \text { at } x_{j}=0 \text { or } L_{j}, \\
j=1,2,3, i=1, \ldots, S .
\end{gathered}
$$

The $N_{i R}$ are defined at all points of $\Omega_{R}$ using the formulas (4.4) written as in the Neumann case. We then solve equations (5.5) at the points $x \in \Omega_{R}^{0}$, calling $K_{i R}$ the corresponding approximations (and $C_{i R}(x)=e^{K_{i R}(x)}, \forall x \in \Omega_{R}$ ). The existence of a solution $K_{i R}$ on an interval of time $\left(0, T_{*}\right), 0<T_{*} \leq T$ is known; the a priori estimate guarantees that $T_{*}=T$. The a priori estimate is obtained as follows: we write the sum

$$
\sum_{i=1}^{S} \sum_{k=1}^{3} \sum_{x \in \Omega_{R}}^{S} \frac{\partial N_{i R}^{(k)}}{\partial x_{k}}(x)\left(K_{i R}(x)-K_{i b}(x)\right)\left(\varpi_{1}^{i_{1}} \varpi_{2}^{i_{2}} \varpi_{3}^{i_{3}}\right)(x),
$$

where $N_{i R}^{(k)}$ is the $k^{\text {th }}$ component of $N_{i R}$, and $x \in \Omega_{R}$ is of the form $x=\left(x_{1}^{i_{2}}, x_{2}^{i_{2}}, x_{3}^{i_{3}}\right)$, $0 \leq i_{1} \leq R_{1}, 0 \leq i_{2} \leq R_{2}, 0 \leq i_{3} \leq R_{3}$. We observe that $K_{i R}-K_{i b}=0$ at $x_{k}^{0}$ and $x_{k}^{R_{k}}$ in each direction; hence the above sum is in fact reduced to the points $x \in \Omega_{R}^{0}$. We will use the sum for $x \in \Omega_{R}^{0}$ in one case and the sum for $x \in \Omega_{R}$ in the second case.

First we use Eq. (5.5) at the points $x \in \Omega_{R}^{0}$ and replace this sum by

$$
\begin{aligned}
& \sum_{i=1}^{S} \sum_{x \in \Omega_{R}^{0}}\left(e^{K_{i R}} \frac{\partial K_{i R}}{\partial t}-\omega_{i}\right)\left(K_{i R}-K_{i t}\right)(x, t) \\
& =\frac{\partial}{\partial t} \Phi_{R}\left(C_{1 R}, \ldots, C_{S R}\right)-\sum_{i=1}^{S} \sum_{x \in \Omega_{R}^{0}}\left(\frac{C_{i R}}{C_{i b}} \frac{\partial C_{i b}}{\partial t}\right)(x) .
\end{aligned}
$$

Here $C_{i_{R}}(x, t)=e^{K_{i R}(x, t)}, \forall x \in \Omega_{R}$, and

$$
\begin{aligned}
& \Phi\left(C_{1 R}, \ldots, C_{S R}\right) \\
& =\sum_{i=1}^{S} \sum_{x \in \Omega_{R}^{0}}\left(C_{i R} \log C_{i R}-C_{i R}+P+C_{i R} \log C_{i b}(x)\right) .
\end{aligned}
$$

As in Sec. 3, the constant $P$ is chosen, such that

$$
\begin{aligned}
& r \log r-r+P+r \log C_{i b}(x) \geq 0, \\
& \forall x \in \Omega, i=1, \ldots, S, \forall r \in \mathbb{R} .
\end{aligned}
$$

Now we see the sum (5.22) differently with $x \in \Omega_{R}$ : since $\partial N_{i R} / \partial x_{k}$ is a polynomial of degree $\leq R_{k}-1$ in $x_{k}$, and $K_{i R}-K_{i b}$ is a polynomial of degree $\leq R_{k}$ in $x_{k}$, the product is of degree $2 R_{k}-1$ at most in $x_{k}$. Hence the quadrature formula is exact in $x_{k}$, $k=1,2,3$, and the sum (5.22) is exactly equal to the integral

$$
\sum_{i=1}^{S} \int_{\Omega} \nabla\left(N_{i R}\right)\left(K_{i R}-I_{R} K_{i b}\right) d x
$$


We integrate by parts, and we find, observing that $K_{i R}-K_{i b}=0$ on $\partial \Omega$,

$$
\sum_{i=1}^{S} \int_{\Omega} N_{i R} \cdot \nabla\left(K_{i R}-I_{R} K_{i b}\right) d x
$$

Observing that the integral is a polynomial of degree $\leq 2 R_{k}-1$ in each variable $x_{k}$, $k=1,2,3$, we can replace again the last quantity by the following sum corresponding to an exact quadrature formula:

$$
\begin{aligned}
& \sum_{i=1}^{S} \sum_{x \in \Omega_{R}}\left[N_{i_{R}}-\nabla\left(K_{i R}-I_{R} K_{i b}\right) \varpi\right](x) \\
& =\left(\text { by equations (4.4) at the } x \in \Omega_{R}\right. \text { ) } \\
& =\sum_{i=1}^{S} \sum_{x \in \Omega_{R}}\left(N_{i R} I_{R}\left(K_{i b}\right) \varpi\right)(x) \\
& -\sum_{i=1}^{S} \sum_{x \in \Omega_{R}}\left\{\left[d_{i} e^{-K_{i R}} N_{i R}\right.\right. \\
& \left.\left.+\frac{\mathcal{R} T}{p} \sum_{\substack{j=1 \\
j \neq i}}^{S} d_{i j} e^{K_{j}}\left(e^{-K_{i}} N_{i_{R}}-e^{-K_{j}} N_{j_{R}}\right)+d_{i} g \nabla p\right] N_{i_{R}} \varpi\right\}(x) \\
& =\sum_{i=1}^{S} \sum_{x \in \Omega_{R}}\left[\left(I_{R}\left(K_{i b}\right)+d_{i} g \nabla p\right) N_{i R} \varpi\right](x) \\
& -\sum_{i=1}^{S} \sum_{x \in \Omega_{R}}\left(d_{i} e^{-K_{i R}} N_{i_{R}}^{2} \varpi\right)(x) \\
& -\frac{1}{2} \sum_{i=1}^{2} \sum_{x \in \Omega_{R}} \frac{\mathcal{R} T}{p} \sum_{\substack{j=1 \\
j \neq i}}^{S}\left[d_{i j} e^{K_{i}+K_{j}}\left(e^{-K_{i}} N_{i R}-e^{-K_{j}} N_{j_{R}}\right)^{2} \varpi\right](x) \\
& \leq \sum_{i=1}^{S} \sum_{x \in \Omega_{R}}\left[\left(I_{R}\left(K_{i b}\right)+d_{i} g \nabla p\right) N_{i R} \varpi\right](x) \\
& -\sum_{i=1}^{S} \sum_{x \in \Omega_{R}}\left(d_{i} e^{-K_{i R}} N_{i R}^{2} \varpi\right)(x) \\
& \leq-\frac{1}{2} \sum_{i=1}^{S} \sum_{x \in \Omega_{R}}\left(d_{i} e^{-K_{i R} N_{i R}^{2} \varpi}\right)(x) \\
& +\frac{1}{2} \sum_{i=1}^{S} \sum_{x \in \Omega_{R}}\left[d_{i}^{-1} e^{K_{i R}}\left(I_{R}\left(K_{i b}\right)+d_{i} g \nabla p\right)^{2} \varpi\right](x) .
\end{aligned}
$$


Hence

$$
\begin{aligned}
& \sum_{i=1}^{S} \sum_{x \in \Omega_{R}}\left[N_{i_{R}}-\nabla\left(K_{i R}-I_{R} K_{i b}\right) \varpi\right](x) \\
& \quad \leq \kappa_{1}^{\prime}+\kappa_{2}^{\prime} \sum_{i=1}^{S} \sum_{x \in \Omega_{R}^{0}} C_{i R}(x),
\end{aligned}
$$

where $\kappa_{1}^{\prime}, \kappa_{2}^{\prime}$ are independent of $x$ and $t$ (and $R$ ), and $C_{i R}(x)=e^{K_{i R}(x)}, x \in \Omega_{R}$. Note that the last sum is for $x \in \Omega_{R}^{0}$, the terms $x \in \Omega_{R} \backslash \Omega_{R}^{0}$ being absorbed by $\kappa_{1}^{\prime}$.

Comparing with (5.23)-(5.24), we find:

$$
\begin{aligned}
& \frac{\partial \Phi_{R}}{\partial t}\left(C_{1 R}, \ldots, C_{S R}\right) \\
& \leq \kappa_{1}^{\prime}+\kappa_{2}^{\prime} \sum_{i=1}^{S} \sum_{x \in \Omega_{R}^{0}} C_{i R}(x)+\sum_{i=1}^{S} \sum_{x \in \Omega_{R}^{0}}\left(\frac{C_{i R}}{C_{i b}} \frac{\partial C_{i b}}{\partial t}\right)(x) \\
& \quad \leq \kappa_{1}^{\prime}+\kappa_{3}^{\prime} \sum_{i=1}^{S} \sum_{x \in \Omega_{R}^{0}} C_{i R}(x) .
\end{aligned}
$$

Increasing $P$ if needed, we observe that there exists a constant $\lambda>0$ such that

$$
\begin{aligned}
& r \leq \lambda\left(r \log r-r+P+r \log C_{i b}(x)\right), \\
& \forall x \in \Omega, i=1, \ldots, S, \forall r \in R .
\end{aligned}
$$

Then (5.25) yields

$$
\frac{\partial \Phi_{R}}{\partial t}\left(C_{1 R}, \ldots, C_{S R}^{\prime}\right) \leq \kappa_{1}^{\prime}+\kappa_{3}^{\prime} \Phi_{R}\left(C_{1 R}, \ldots, C_{S R}\right)
$$

From (5.26) we deduce that the solution to the approximate system is defined for $0 \leq t \leq T$, and that it is bounded on this interval as follows:

$$
\begin{aligned}
\sum_{i=1}^{S} \sum_{x \in \Omega_{R}^{0}} C_{i R}(x, t) & \leq \lambda^{-1} \Phi\left(C_{1 R}, \ldots, C_{S R}\right)(t) \\
& \leq \lambda^{-1}\left[\Phi\left(C_{1 R}, \ldots, C_{S R}\right)(0)+\frac{\kappa_{1}^{\prime}}{\kappa_{3}^{\prime}}\right] e^{\kappa_{3} T} .
\end{aligned}
$$

The stability of the scheme is established in this case as well (namely semi-discretization in space by pseudo-spectral method in the case of the nonhomogeneous-Dirichlet boundary condition).

REMARK 5.2. Numerical simulations and a more detailed analysis in the one-dimensional case will appear in a subsequent paper [3].

Acknowledgements. This work was partially supported by the Department of Energy under the grant DE-FG02-98-ER25346, by the National Science Foundation under the grant NSF-DMS-0074334, and by the Research Fund of Indiana University. 


\section{REFERENCES}

[1] R. Aris, The Mathematical Theory of the Diffusion and Reaction in Permeable Catalysts, Oxford University Press, London, 1975

[2] H. C. Chang, D. Gottlieb, M. Marion, and B. W. Sheldon, Mathematical Analysis and Optimization of Infiltration Processes, Journal of Scientific Computing, Vol. 13, No. 3, 1998

[3] A. Ditkowski, D. Gottlieb, and R. Temam, article in preparation

[4] A. Ern and V. Giovangigli, Multicomponent Transport Algorithms, Lecture Notes in Physics, Springer-Verlag 24, 1994

[5] D. Funaro, Spectral elements for transport-dominated equations, Lecture Notes in Computational Science and Engineering, 1, Springer-Verlag, Berlin, 1997

[6] D. Funaro, Polynomial approximation of differential equations, Lecture Notes in Physics, New Series $\mathrm{m}$ : Monographs, 8, Springer-Verlag, Berlin, 1992

[7] D. Gottlieb and S. A. Orszag, Numerical analysis of spectral methods: theory and applications, CBMS-NSF Regional Conference Series in Applied Mathematics, No. 26, Society for Industrial and Applied Mathematics, Philadelphia, Pa., 1977

[8] R. Jackson, Transport in Porous Catalysts, Elsevier Science Publisher, 1977

[9] F. A. Williams, Lectures on applied mathematics in combustion. Past contributions and future problems in laminar and turbulent combustion, Phys. D 20, no. 1, 21-34 (1986) 